\title{
Minor Stroke and Thrombolysis: What Is in the Pipeline?
}

W e read with interest the article by Messer et $\mathrm{al}^{1}$ regarding the clinical outcome after IV thrombolysis and/or mechanical thrombectomy in patients with acute ischemic stroke due to large-vessel occlusion, presenting as minor stroke syndrome (MSS) (NIHSS score $<5$ ). The authors retrospectively analyzed a group of 378 patients with MSS involving the anterior circulation, finding that $12 \%$ of these had large-vessel occlusion. In this subgroup, they noted that patients with immediate mechanical thrombectomy had a better outcome than patients with delayed thrombectomy (performed after neurologic deterioration) or with IV thrombolysis only. Most interesting, a recent study has shown that almost $30 \%$ of patients not treated with IV thrombolysis because they were initially considered "to-good-to-treat" (NIHSS score < 5) could suddenly worsen during the first hours following the stroke onset. This finding suggests possibly including this subgroup of patients in the IV thrombolysis decisionmaking algorithms. ${ }^{2}$ Mechanical thrombectomy has become the standard of care for patients presenting with severe ischemic stroke due to large-vessel occlusion; however, it is unclear whether patients presenting with MSS would benefit from this treatment.

We have retrospectively analyzed a group of 104 patients admitted to our institution (Misericordia Hospital, Grosseto) for acute ischemic stroke during 2015-2016 who received standard IV thrombolysis. We identified 21 patients (22\%) with MSS. Thirteen of those 21 patients $(62 \%)$ had a good outcome at discharge $(\mathrm{mRS}<2)$. No symptomatic intracranial hemorrhage occurred. All patients underwent CTA. Eleven of those 21 patients had large-vessel occlusion. Specifically, 8 patients (38\%) had largevessel occlusion in the anterior circulation (respectively, 2 patients with ICA extracranial occlusion, and 2 patients with MCA-M1 and 4 patients with MCA-M2 occlusion) and 3 patients $(14 \%)$ had a posterior circulation stroke (1 patient with basilar artery apex occlusion and 2 patients with extracranial vertebral artery occlusion). One patient had an extracranial ICA dissecting pseudoaneurysm without large-vessel occlusion. Four of these 11 patients were initially treated with mechanical thrombectomy according to the model of "drip and ship," with good out-

http://dx.doi.org/10.3174/ajnr.A5298 come at discharge $(\mathrm{mRS}<2)$. Seven of these 11 patients were treated with IV thrombolysis only. Among this latter subgroup, 4 patients had early neurologic deterioration, and no mechanical thrombectomy was performed later because too much time had elapsed. Moreover, the patient with an ICA dissecting pseudoaneurysm had an early relapse due to an embolism.

Although our case series is too small to clarify the potential benefit of mechanical thrombectomy in MSS, this retrospective observation caused some reflection. First, we confirm that a relevant percentage of large-vessel occlusion in MSS involves not only the anterior but also the posterior circulation (52\% of our observed cases). The management of MSS is currently unclear, and we would like to stress that the NIHSS alone does not represent a valid outcome predictor or an indicator of large-vessel occlusion in this subgroup of patients. In fact, a posterior circulation stroke might initially present with a low NIHSS score, as previously stated. ${ }^{2,3}$ Again, a low NIHSS score at stroke onset might possibly reflect an anterior circulation stroke due to large-vessel occlusion in the presence of valid collateral circulation. These conditions might worsen in the immediate hours following.

We agree with Messer et $\mathrm{al}^{1}$ that an advanced neuroimaging diagnostic work-up, including CTA, is suggested in MSS to correctly address the treatment. Second, in case of large-vessel occlusion (involving the anterior or posterior circulation), an immediate evaluation to the "hub" center for mechanical thrombectomy should be considered, given that this condition is the most common cause of early neurologic deterioration after IV thrombolysis in MSS. We believe that "spoke" centers cannot wait for neurologic deterioration to transfer the patient to the referring "hub"; in this case, the patient cannot undergo mechanical thrombectomy because of elapsed time. Third, an early severe stroke relapse may erase the benefit of IV thrombolysis when a high-risk embolic condition is present (eg, a severe ICA stenosis/dissection) as previously reported for TIAs. Thus, we recently admitted a man 63 years of age presenting with MSS due to an ICA dissecting pseudoaneurysm that relapsed a few hours after IV thrombolysis into a more disabling stroke. In this case, as suggested by Behme et al, ${ }^{4}$ a patient having undergone thrombolysis with a high-grade extracranial ICA stenosis should be considered for ICA stent placement immediately after mechanical thrombectomy. On the other hand, the best management for patients without tandem lesions is 
still unclear. Do we need to transfer the patient, according to the drip and ship model, to the referring ring hub for acute stent placement? Alternatively, is it preferable to perform mechanical thrombectomy only after the occurrence of an early relapse?

In conclusion, the correct management of MSS is unclear. While one waits for the results of ongoing clinical trials in this subgroup of patients (PRISMS, NCT02072226; clinicaltrials.gov), the experience of single-center studies might help clinicians to better define the correct therapeutic algorithms.

\section{REFERENCES}

1. Messer MP, Schönenberger S, Möhlenbruck MA, et al. Minor stroke syndromes in large-vessel occlusions: mechanical thrombectomy or thrombolysis only? AJNR Am J Neuroradiol 2017;38:1177-79 CrossRef Medline

2. Ali SF, Siddiqui $\mathrm{K}, \mathrm{Ay} \mathrm{H}$, et al. Baseline predictors of poor outcome in patients too good to treat with intravenous thrombolysis. Stroke 2016;47:2986-92 CrossRef Medline

3. Kim JT, Park MS, Choi KH, et al. Clinical outcomes of posterior versus anterior circulation infarction with low National Institutes of Health Stroke Scale scores. Stroke 2017;48:55-62 CrossRef Medline

4. Behme D, Knauth M, Psychogios MN. Retriever wire supported carotid artery revascularization (ReWiSed CARe) in acute ischemic stroke with underlying tandem occlusion caused by an internal carotid artery dissection: technical note. Interv Neuroradiol 2017;23: 289-92 CrossRef Medline

(D) Gallerini

Unit of Neurology Misericordia Hospital Grosseto, Italy (D). Marsili Unit of Neurology Misericordia Hospital Grosseto, Italy Department of Neurology and Psychiatry "Sapienza" University of Rome Rome, Italy (D) M. Bartalucci

(i) C. Marotti (D). Marconi Unit of Neurology Misericordia Hospital Grosseto, Italy 